\title{
Reflections on the Design and Application of Modern Chinese Characters
}

\author{
Bing Liu 1, a, Ruiqiu Pang 2, * \\ ${ }^{1}$ Academy of Fine Arts, Northeast Normal University, Changchun130024, China \\ 2 School of Geographical Sciences, Northeast Normal University, Changchun130024, China \\ a ice8515@163.com
}

Keywords: Chinese characters; Implication; Graphic; Image beauty.

Abstract. This article focuses on the expression of the image and artistic conception of Chinese characters; the design and application, the opportunities and challenges of Chinese characters elements in modern design are analyzed. Chinese character as a pictograph, it is the inner artistic conception of "implication", and the organic combination of identity, artistry and integrity on "graphic", and has a unique "image beauty", it embodies the profound cultural connotation and unique artistic charm, permeate Chinese people's subjective aesthetic emotion and value judgment; multimedia and digitization are the main factors that influence the development of Chinese character art design, under the guidance of modern design concept, relying on the highly developed science and technology and the modern spirit of the times, give full play to the practical value and design potential of Chinese characters, it is of great significance to study the artistic design of Chinese characters.

\section{Introduction}

Chinese characters are rich in cultural information, it is the only hieroglyphic system still in use in the world, and it is also a kind of graphic and symbolic text with rich connotation and strong expressive force, building the way we perceive the world, however, under the background of international development, the design and application of Chinese characters have been greatly impacted and challenged. Chinese character is a pictograph, after thousands of years of evolution and development, and in the long history, and gradually formed a shape ideographic, intended to convey the font structure. Rudolf Arnheim believes that in all areas of human activity, the real creative thinking is carried out through the image [1-3].

Chinese character as a pictograph, it is embodied in the simplification, generalization and materialization of the characteristics of things, it is the result of Chinese people's long-term observation and creation, inheritance and accumulation of social culture, it also embodies the profound cultural connotation and unique artistic charm. Chinese characters penetrate Chinese subjective aesthetic emotion and value judgment, it is the crystallization of Chinese cultural wisdom, has a very special visual communication. Chinese characters, as a faithful record of Chinese civilization, and a solid carrier of Chinese culture, the cultural connotation of which is broad and profound $[4,5]$.

\section{The Unique Design Effect of Chinese Characters}

\section{"Implication" and "Graphic" of Chinese Characters.}

At the beginning of creation, Chinese characters embody the combination of "implication" and "graphic". The design of Chinese characters is to decorate and change on the basis of traditional fonts, it is the extension of the pictographic form in the external form, and divorced from the basic glyph and stroke constraints, according to the content of the text, the use of rich imagination and formal beauty, constitute the font of Chinese characters [6].

\footnotetext{
* Corresponding author: Ruiqiu Pang, Associate professor, Northeast Normal University, CHINA.
} 
Design makes Chinese characters more vivid. Chinese characters as an important element in visual communication, is not only a kind of decoration, should also be based on the content to convey its rich cultural implications, this is also an important feature of Chinese characters different from the Latin alphabet.

The "graphic" and "implication" of Chinese character design can be regarded as the unity of form and content, it not only considers the transmission of information, but also reflects the aesthetic nature of art. So, "graphic" of Chinese characters is the design of the external visual charm, and "implication" is a rich and thought-provoking cultural implication, only the perfect combination of graphics and implication can deduce the beauty of the form of Chinese character design. The organic combination of "graphic" and "implication" in the Chinese character design, according to the audience's visual perception, aesthetic concept, the actual application of the needs of the development and change, and into the unique form and aesthetic concept, to highlight the formal beauty of Chinese character design. Hearing from the "graphic" of the association, the perception of "implication" charm, that is the deepening and refining of "form" and "meaning", it is the conception and design of "graphic" and "implication".

\section{"Image Beauty" of Chinese Characters}

The structure of Chinese characters makes it have unique "image beauty", mainly reflected in the local, the pen shape, structure and the overall design of the grasp, is a point, line layout of visual art, stable balance shows the character of different fonts. The text is composed of horizontal, vertical, point and arc lines, in the arrangement of the structure and the collocation of the line, only coordinate the relationship between strokes and words, emphasize the rhythm of font strokes, can better to display the font art, this is not only clear the characteristics of the text, and enrich the connotation of the font, create a strong artistic appeal of the font design works.

"Image beauty" of Chinese characters, embodied in the form of text and aesthetic characteristics of the integration process, the implication of beauty, because of the different fields of application, they have different attitudes, modern Chinese character art, like other modern art, in essence, it is the creation of ideology, and it is a kind of change in concept. Visual features of Chinese characters, the Chinese characters from the word meaning communication to achieve graphic expression, and it has strong plasticity, in the form of expression, the integration of decoration, design and many other factors, creation of conceptual works such as Chinese character device, visual communication design, behavior art, sculpture and so on, and these works are closely related to the use of the aesthetic features of Chinese characters.

\section{The Opportunity and Challenge of Chinese Characters in Modern Design}

The evolution of Chinese characters, it can be seen as a long and complex design process, this process continues until today. Faced with the arrival of the information age, many of the traditional text technology has been a serious impact and lag, Chinese characters due to the emergence of a series of coding technology, to be able to enter the computer font, Greatly accelerated the process of font design. The arrival of the keyboard era, there is no need to worry about the speed of writing, and pay more attention to Chinese characters to convey meaning, as a design element, the Chinese character is applied to all kinds of art forms. It can be seen that digitization provides opportunities for the prosperity and development of Chinese character design, but the convenience of digital also leads to the over reliance on computers, the traditional writing and calligraphy art is gradually replaced by the keyboard, often, the pen to forget the word phenomenon. Young designers rarely practice and write basic fonts, leave the computer, you cannot design fonts, this dependence makes designers lack of understanding of Chinese characters and calligraphy, resulting in the design of Chinese characters without vitality.

In the new media era, for computers, mobile phones, movies, television and other media, the combination of Chinese characters and multimedia, multi dimensional performance and development of Chinese characters, not only in the visual innovation, it also gives us a fresh experience of hearing, 
touch and smell. No matter how innovative, our aim is to tap the potential strength of Chinese characters, to explore the local culture of the nation, to find the collision point between traditional culture and their own personality, let young people feel the charm of Chinese characters, let the Chinese elements and Chinese culture have a unique influence on the world.

In recent years, there are many famous designers in the design field to make a lot of excellent works of Chinese characters, in different fields and carriers, the innovative design of the Chinese character elements reflects the enormous vitality and appeal, thus causing the Chinese characters as the theme of the design boom, this upsurge has played a positive role in the development of modern Chinese characters and the study of modern visual language of Chinese characters, as a carrier of Chinese culture, Chinese characters have gained greater power in the world.

The design of Chinese characters is a new idea and method in the field of design in recent years, Today's rapid development of social and cultural patterns, economic activities, scientific and technological conditions and information dissemination media, to promote the continuous evolution of Chinese characters art design; The function, form and aesthetics of Chinese characters, but also appeared new concepts and techniques. Chinese characters are used as the modeling elements in modern design, has irreplaceable design effect, fully demonstrated its great artistic charm and cultural heritage. Give full play to the practical value and design potential of Chinese characters, to enhance the visual impact of Chinese characters, to study the art design form of Chinese characters, significant meaning. Facing the globalization of design, under the guidance of modern design concept, relying on the highly developed science and technology and the modern spirit of the times, Chinese characters and the combination of Chinese and Western art design will shine.

\section{Summary}

Chinese character as a pictograph, embodied in it is the simplification of the characteristics of things, generalization and specialization, it is the result of Chinese people's long-term observation and creation, not only inherit and accumulate social culture, it also embodies the profound cultural connotation and unique artistic charm. Chinese characters penetrate Chinese subjective aesthetic emotion and value judgment, it is the crystallization of Chinese cultural wisdom, has very special visual communication characteristics, the high degree of symbolization and systematization makes it have irreplaceable function effect of other elements, and has a strong cultural appeal. By virtue of the unique visual situation on Chinese characters, through innovative visual form, showing a "meaningful form", make full use of the rich and powerful vitality of Chinese characters, communicate with the audience directly and effectively, Chinese characters will become a bridge of cultural exchange and communication with the world, effectively promote the development of Chinese culture in the era of the forefront of the world.

\section{Acknowledgements}

The authors gratefully thank the financial support by Research Project in Jilin Social Science Fund Project (2013BS14); Youth fund of Northeast Normal University in philosophy and social science school projects-art project (MSZX2012-01).

\section{Corresponding author}

Ruiqiu Pang, Associate professor, School of Geographical Sciences, Northeast Normal University, Changchun130024, CHINA.

\section{References}

[1]. Chia-Fen Chi, Dengchuan Cai, Manlai You, Applying image descriptors to the assessment of legibility in Chinese characters, Ergonomics, vol. 46(8), 2003, pp. 825-841. 
[2]. Basile Zimmermann, Redesigning Culture: Chinese Characters in Alphabet-Encoded Networks, Design and Culture, vol. 2(1), 2010, pp. 27-43.

[3]. Shouhui Zhao, Chinese Character Modernisation in the Digital Era: A Historical Perspective, Current Issues in Language Planning, vol. 6(3), 2005, pp. 315-378.

[4]. Mengmeng Zhou, Jiao Meng, The Integration Between Chinese Characters Design and National Culture, The International Conference on Education, Language, Art and Intercultural Communication (ICELAIC), 2014, 519-521.

[5]. Xinghai Chen, Huan Yang, Haijin Liao, Thinking of the Future Attribute of Chinese Characters Under the Condition of New Media Technology, E-Business Creative Design Manufacturing - 2009 IEEE 10th International Conference on Computer-Aided Industrial Design \& Conceptual Design (CAID\&CD) , 2009, 1914-1917.

[6]. Wang Ruiming, Chinese Character Design Presented in Pictograph and Symbolism at the Beginning of Signifying Search, 3rd International Conference on Education and Management Technology (ICEMT), 2012, 243-247. 